\title{
Dose-related effects of propericiazine in rats
}

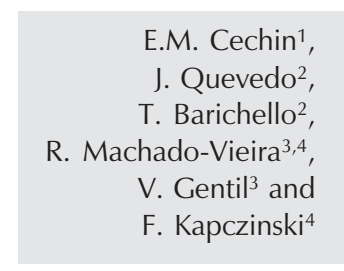

\section{Correspondence \\ F. Kapczinski \\ Rua Costa, 30/501 \\ 90110-270 Porto Alegre, RS \\ Brazil \\ Fax: +55-51-3232-3766 \\ E-mail: kapcz@zaz.com.br}

Research supported by FAPERGS, PRONEX, PROPESQ/UFRGS, FIPE/ HCPA, and CESP, Projeto Regulação do Humor. Publication supported by FAPESP.

Received January 15, 2002

Accepted October 3, 2002

$\ldots \ldots \ldots \ldots \ldots \ldots \ldots$

\author{
${ }^{1}$ Grupo de Neurociências, Departamento de Medicina Interna, \\ Faculdade de Medicina, Universidade de Passo Fundo, Passo Fundo, RS, Brasil \\ ${ }^{2}$ Laboratório de Farmacologia, Departamento de Medicina, \\ Universidade do Extremo Sul Catarinense, Criciúma, SC, Brasil \\ ${ }^{3}$ Departamento de Psiquiatria, Faculdade de Medicina, Universidade de São Paulo, \\ São Paulo, SP, Brasil \\ ${ }^{4}$ Grupo de Psicofarmacologia, Serviço de Psiquiatria, Hospital de Clínicas de Porto \\ Alegre e Departamento de Psiquiatria, Faculdade de Medicina, Universidade Federal \\ do Rio Grande do Sul, Porto Alegre, RS, Brasil
}

\section{Abstract}

We evaluated the effects of the neuroleptic agent propericiazine on animal models of anxiety and memory. Adult male Wistar rats (250 to $350 \mathrm{~g})$ received intraperitoneal injections of propericiazine $(0.05$, 0.075 and $0.1 \mathrm{mg} / \mathrm{kg}$ ), diazepam $(1 \mathrm{mg} / \mathrm{kg})$, saline, or diazepam vehicle (20\% propylene glycol and $80 \%$ saline) 30 min prior to the experimental procedure. Animals (10-15 for each task) were tested for step-down inhibitory avoidance (0.3-mA footshock) and habituation to an open-field for memory assessment, and submitted to the elevated plus-maze to evaluate the effects of propericiazine in a model of anxiety. Animals treated with $0.075 \mathrm{mg} / \mathrm{kg}$ propericiazine showed a reduction in anxiety measures $(\mathrm{P}<0.05)$ similar to that observed in those treated with diazepam. Propericiazine at the doses of 0.05 and $0.1 \mathrm{mg} / \mathrm{kg}$ had no significant anxiolytic effects $(\mathrm{P}>0.05)$ in the elevated plus-maze model of anxiety. Memory was not affected by propericiazine in any of the tests, but was impaired by diazepam. The results indicate a dose-related, inverse U-shaped effect of propericiazine in an anxiety model, but not on memory tasks, perhaps reflecting involvement of the dopaminergic system in the mechanisms of anxiety.
Propericiazine, a piperidine phenothiazine similar to thioridazine, was introduced for the treatment of psychosis in the early sixties $(1,2)$, with particular emphasis on its "usefulness in the control of aggressive manifestations". The literature on this drug is limited to 74 articles published between 1965 and May 2001 (MEDLINE/PubMed), the last one in 1996. No reliable information on its dose-equivalence in terms of clinical efficacy or adverse effects compared with other psychotropic drugs is available.

Initial studies indicated that propericia-
Key words - Propericiazine

- Memory

- Anxiety

- Diazepam

- Elevated plus-maze zine was less effective than chlordiazepoxide for the treatment of anxiety (2). This, however, was never studied in a systematic manner under the conditions in which clinicians used this medication for almost 40 years in many countries. Indeed, its package insert in Brazil recommends propericiazine for "character and behavioral disorders", and suggests that it is particularly indicated for conditions such as autism, impulsiveness, hostility, irritability, aggressiveness, reactions to frustration, psychomotor and emotional instability, among others, at average doses of 
20-25 mg/day (Package Insert for Neuleptil ${ }^{\circledR}$, Rhodia Farma, São Paulo, SP, Brazil). Clinical lore supports its use as chief or adjuvant therapy for anxiety in old age, and irritability and tension in late luteal-phase dysphoria, obsessive-compulsive disorder, and temporal lobe epilepsy. Again, it is particularly interesting that the dose often prescribed by clinicians is so low (less than $10 \mathrm{mg} /$ day) as to preclude extrapolations from what is known about the mechanism of action of the drug from preclinical studies in which much higher doses were used. For instance, it is possible that propericiazine's clinical effects occur without strong dopamine receptor blockade.

Given its claimed effectiveness in troubled children and adolescents $(3,4)$ and for aggressiveness in dementia at doses of up to 40 $\mathrm{mg} /$ day (5) and the traditional adjuvant use mentioned above, together with its low cost, particularly at low doses, a reevaluation of the potential merits and mechanisms of action of propericiazine is justified.

Preclinical studies have suggested an overall pharmacological similarity of propericiazine with chlorpromazine (2). Dopamine receptor subtype analysis has not been performed for propericiazine, but the drug appears to induce greater noradrenergic than dopaminergic blockade (6). Compared to chlorpromazine, propericiazine reportedly has more potent antiemetic, antiserotonin, and anticholinergic activity $(7,8)$. More potent cataleptic activity compared to prochlorperazine has been demonstrated (8). In mice, the toxicity of oral propericiazine has been less than that of oral chlorpromazine (9). Propericiazine may possess mild stimulant properties (2).

Several lines of evidence suggest that noradrenergic and dopaminergic mechanisms have a modulatory role in emotional behavior and anxiety (8). Early animal studies reported anticonflict effects for dopamine receptor antagonists (8), whereas clinically, small doses of chlorpromazine and haloperidol can be as effective as benzodiazepines in the management of anxiety disorders (10).

The objective of the present study was to assess the effects of very low doses of propericiazine on a model of anxiety in rats using the elevated plus-maze model. The elevated plus-maze has been validated for both rats and mice $(8,11)$, and is sensitive to both increases and decreases in behavior associated with anxiety (11). In addition, we evaluated the effects of propericiazine on two memory tests, i.e., habituation to an open-field (12) and step-down inhibitory avoidance task (13) in order to distinguish the effect on memory of propericiazine from that of the benzodiazepines.

Adult male Wistar rats weighing 250 to $350 \mathrm{~g}$ were used. The animals were housed in plastic cages ( $40 \times 30 \times 15 \mathrm{~cm})$, five per cage, under a 12-h light/dark cycle (lights on at 7:00 am) at a constant temperature of $23 \pm$ $1^{\circ} \mathrm{C}$ with free access to food and tap water. Different rats were used for each of the three behavioral tasks ( $\mathrm{N}=10$ to 15$)$. The tests were conduced between 2:00 and 5:00 pm.

Propericiazine (Neuleptil ${ }^{\circledR}$, Rhodia Farma) at doses of $0.05,0.075$ and $0.1 \mathrm{mg} / \mathrm{kg}$ (equivalent to 3 to $6 \mathrm{mg} /$ day for a patient of $60 \mathrm{~kg}$ body weight) was dissolved in saline and used immediately. Diazepam (1 mg/kg, Valium $^{\circledR}$, Roche, São Paulo, SP, Brazil) was dissolved in vehicle (20\% propylene glycol and $80 \%$ saline). The injections were given intraperitoneally ( $1 \mathrm{ml} / \mathrm{kg}$ body weight) 30 min before each behavioral task.

The elevated plus-maze test used in animal models of anxiety is described in detail elsewhere $(10,11)$. Briefly, the apparatus consisted of two open arms $(50 \times 10 \mathrm{~cm})$ and two enclosed arms $(50 \times 10 \times 40 \mathrm{~cm})$ arranged in such a way that the two arms of each type were opposite to each other, and a central platform $(5 \times 5 \mathrm{~cm})$. The maze's height was $50 \mathrm{~cm}$ and the tests were conducted under dim red light. Animals were allowed a 5-min exposure to red light in their 
own home cages before the testing procedure. Next, they were placed individually on the central platform of the plus-maze facing an open arm. During a 5-min test period, the following measurements were recorded by two observers: the number of entries and the time spent in the open and closed arms and the total number of arm entries.

In the habituation to an open field, animals were exposed twice, with a 24-h interval, to a $40 \times 50 \times 60 \mathrm{~cm}$ open field whose brown linoleum floor was divided into 12 equal squares by black lines. In both sessions, the animals were placed in the rear left square and allowed to explore freely for 5 min, during which time the number of line crossings and rearing responses was recorded (12).

The step-down inhibitory avoidance apparatus consisted of a $50 \times 25 \times 25 \mathrm{~cm}$ plastic box with a front glass wall, whose floor had parallel 10-mm bronze bars. The left end of the grid was occupied by a 7-cm wide, 2.5 $\mathrm{cm}$ high Formica platform. The rats were gently placed on the platform facing the rear wall, and their latency to step down with all four paws on the grid was measured. In the training session, after stepping down, the animals received a $0.3-\mathrm{mA}, 2-\mathrm{s}$ scrambled footshock, and were withdrawn immediately from the cage. In the test session, $24 \mathrm{~h}$ later, the procedure was repeated, but the footshock was not given. Test session step-down latency was used as a measure of retention. A ceiling of $180 \mathrm{~s}$ was imposed on this measure, i.e., animals whose test latency was more than $180 \mathrm{~s}$ were considered to have a latency of $180 \mathrm{~s}$ (13).

The data from the elevated plus-maze and open-field are reported as means \pm SEM and were analyzed by one-way ANOVA followed by the Duncan test. In the openfield, the differences between training and test sessions were analyzed by the Student $t$ test. The analysis of the data obtained in the step-down inhibitory avoidance task was nonparametric because this procedure involved a cutoff score. The data are reported as medians (interquartile ranges) and were analyzed by the Kruskal-Wallis test followed by the Mann-Whitney test when necessary. Training-test differences were evaluated by the Wilcoxon test. $\mathrm{P}$ values less than 0.05 were considered to be statistically significant.

The ip administration of $0.075 \mathrm{mg} / \mathrm{kg}$ propericiazine $30 \mathrm{~min}$ before the test produced an anxiolytic-like action in the elevated plus-maze, as indicated by an increase in parameters related to the open arms and a decrease in parameters related to the closed arms of the maze $(\mathrm{P}<0.05)$. Table 1

\begin{tabular}{|c|c|c|c|c|c|}
\hline \multirow[t]{2}{*}{ Group } & \multicolumn{2}{|c|}{ Open arms } & \multicolumn{2}{|c|}{ Closed arms } & \multirow{2}{*}{ 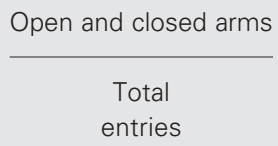 } \\
\hline & $\begin{array}{c}\text { Number of } \\
\text { entries }\end{array}$ & $\begin{array}{c}\text { Time } \\
\text { spent (s) }\end{array}$ & $\begin{array}{c}\text { Number of } \\
\text { entries }\end{array}$ & $\begin{array}{c}\text { Time } \\
\text { spent (s) }\end{array}$ & \\
\hline Saline & $3.5 \pm 0.6$ & $64.1 \pm 10.1$ & $6.7 \pm 0.9$ & $201.0 \pm 13.1$ & $10.3 \pm 1.3$ \\
\hline Control & $4.0 \pm 0.7$ & $71.6 \pm 15.1$ & $7.0 \pm 1.2$ & $197.6 \pm 17.3$ & $11.0 \pm 2.0$ \\
\hline Diazepam & $7.1 \pm 0.7^{*}$ & $183.9 \pm 22.4^{*}$ & $2.5 \pm 0.4^{*}$ & $94.4 \pm 22.7^{*}$ & $9.6 \pm 0.9$ \\
\hline \multicolumn{6}{|l|}{ Propericiazine } \\
\hline $0.05 \mathrm{mg} / \mathrm{kg}$ & $3.7 \pm 0.6$ & $66.2 \pm 11.0$ & $6.8 \pm 0.9$ & $202.9 \pm 13.0$ & $10.5 \pm 1.5$ \\
\hline $0.075 \mathrm{mg} / \mathrm{kg}$ & $6.0 \pm 0.5^{*}$ & $155.4 \pm 9.1^{*}$ & $4.8 \pm 0.3^{*}$ & $101.2 \pm 7.8^{*}$ & $10.9 \pm 0.5$ \\
\hline $0.1 \mathrm{mg} / \mathrm{kg}$ & $3.5 \pm 0.6$ & $65.8 \pm 11.7$ & $6.6 \pm 1.0$ & $202.6 \pm 15.0$ & $10.1 \pm 1.5$ \\
\hline
\end{tabular}

Rats received $0.05,0.075$ or $0.1 \mathrm{mg} / \mathrm{kg}$ propericiazine or $1.0 \mathrm{mg} / \mathrm{kg}$ diazepam $1 \mathrm{~h}$ before testing. Data are reported as means \pm SEM. $N=10-15$ animals per group.

${ }^{*} \mathrm{P}<0.05$ compared to saline and control groups (Mann-Whitney test). 
shows the results of the plus-maze test. Diazepam also presented this anxiolytic pharmacological profile $(\mathrm{P}<0.05)$. Neither 0.05 $\mathrm{mg} / \mathrm{kg}$ propericiazine nor $0.1 \mathrm{mg} / \mathrm{kg}$ presented anxiolytic effects. There were no differences in the total number of arm entries between groups. These results show that propericiazine presents an inverse U-shaped dose-response curve for anxiolytic-like effects, because only the intermediate propericiazine dose $(0.075 \mathrm{mg} / \mathrm{kg})$ showed effects on anxiety in the elevated plus-maze.

There were no differences among groups in the inhibitory avoidance training session. In the test session, there were significant differences $(\mathrm{P}<0.05)$ between diazepaminjected animals $(1 \mathrm{mg} / \mathrm{kg})$ and animals that received propericiazine $(0.05,0.075$ and 0.01 $\mathrm{mg} / \mathrm{kg}$ ). Animals treated with diazepam did not show differences in the training or test latencies, demonstrating the amnestic effect of this drug. The group treated with propericiazine at doses of $0.05,0.075$ and $0.1 \mathrm{mg} /$ $\mathrm{kg}$, on the other hand, showed a significant increase in the latency to step down in the test session when compared with the training session $(\mathrm{P}<0.05)$, indicating that memory was acquired for this task. The same behavior was observed for the saline $(\mathrm{P}<0.05)$ and vehicle groups $(\mathrm{P}<0.05)$. These results show

Table 2. Effect of pretreatment with propericiazine on habituation to an open-field.

\begin{tabular}{lcccrr}
\hline \multirow{2}{*}{ Group } & \multicolumn{2}{c}{ Crossings } & & \multicolumn{2}{c}{ Rearings } \\
\cline { 2 - 3 } \cline { 6 - 6 } & Training & Test & & Training & Test \\
\hline Saline & $59.7 \pm 4.3$ & $30.7 \pm 5.5$ & & $25.9 \pm 2.0$ & $11.0 \pm 1.5$ \\
Vehicle & $61.4 \pm 9.2$ & $26.1 \pm 4.1$ & & $26.1 \pm 2.8$ & $12.5 \pm 1.5$ \\
Diazepam & $63.9 \pm 8.0$ & $55.3 \pm 7.2^{*}$ & & $24.2 \pm 3.3$ & $23.4 \pm 2.1^{*}$ \\
Propericiazine & & & & & \\
$\quad 0.05 \mathrm{mg} / \mathrm{kg}$ & $74.3 \pm 7.4$ & $32.0 \pm 4.6$ & & $23.2 \pm 2.6$ & $12.0 \pm 1.7$ \\
$0.075 \mathrm{mg} / \mathrm{kg}$ & $61.7 \pm 6.7$ & $23.5 \pm 4.7$ & & $22.5 \pm 2.5$ & $7.8 \pm 1.6$ \\
$0.1 \mathrm{mg} / \mathrm{kg}$ & $56.7 \pm 7.1$ & $33.8 \pm 6.9$ & & $16.5 \pm 2.3$ & $12.0 \pm 2.3$ \\
& & & & &
\end{tabular}

Rats received $0.05,0.075$ or $0.1 \mathrm{mg} / \mathrm{kg}$ propericiazine or $1.0 \mathrm{mg} / \mathrm{kg}$ diazepam $1 \mathrm{~h}$ before testing. Data are reported as means \pm SEM. $N=10-15$ animals per group. ${ }^{*} \mathrm{P}<0.05$ compared to saline and control groups (one-way ANOVA).

Note: all groups except diazepam presented training-test differences $(P<0.05$, paired $t$ test). that the ip administration of propericiazine to rats $30 \mathrm{~min}$ before the training session for step-down inhibitory avoidance does not induce any effect on memory for this task.

There were no differences in the number of crossings and rearings among the groups in the habituation to the open-field training session (Table 2). In the test session, there was a significant reduction in both crossings and rearings of the diazepam-injected animals $(1 \mathrm{mg} / \mathrm{kg})$ compared to the other groups. The animals injected with $1 \mathrm{mg} / \mathrm{kg}$ diazepam also did not show differences in the number of crossings and rearings between training and test session, demonstrating the amnestic effect of this drug. On the other hand, animals treated with propericiazine at doses of $0.05,0.075$ and $0.1 \mathrm{mg} / \mathrm{kg}$ showed a decreased number of crossings $(\mathrm{P}<0.05)$ and rearings $(\mathrm{P}<0.05)$, indicating habituation to the task environment. A similar behavior was observed for the saline and vehicle groups.

This study employed animal models of learning to assess the effects of the equivalents of small clinical doses of propericiazine on the behavior of rats in the elevated plus-maze, habituation to an open-field and step-down inhibitory avoidance task. The results showed that a $0.075 \mathrm{mg} / \mathrm{kg}$ dose, but not lower $(0.05 \mathrm{mg} / \mathrm{kg})$ or higher $(0.1 \mathrm{mg} / \mathrm{kg})$ doses induced 'anxiolytic' effects on the elevated plus-maze test.

Recent studies have suggested that, as a result of the blockade of dopamine receptors, neuroleptics may be able to influence the sensitivity of other neurotransmittermediated synaptic responses, in particular those mediated by GABA (14): 1) dopamine function within the nigrostriatal, mesolimbic and mesocortical systems is intimately associated with changes in GABA function within these areas, 2) neuroleptic-sensitive, GABAergic projections link these three major dopamine-containing areas, and 3) GABAergic projections from the substantia nigra mediate many of the behavioral phe- 
nomena associated with an increase or decrease in striatal dopaminergic function. Marco et al. (15) have shown that both typical and atypical neuroleptics share the ability to enhance GABA utilization within the nucleus accumbens after chronic administration, and suggested that this common action may be relevant to, or indicative of, antischizophrenic activity. The fact that propericiazine failed to induce any change in memory assessed by habituation to an openfield and a step-down inhibitory avoidance task suggests that this is not mediated by GABA/benzodiazepine systems.

The extrapyramidal side effects of traditional neuroleptic antipsychotics prevent their widespread use in disorders other than schizophrenia. Thioridazine and propericiazine, the aliphatic phenothiazines, and sulpiride are among the exceptions. The comparative roles of anticholinergic and dopaminergic blockade contributions to their better tolerability in the treatment of abnormal mood and anxiety states must be reassessed. A cost-benefit analysis contrasting some of those drugs with the more recently introduced antipsychotics would also be worthy of consideration. Further studies on the pharmacological actions of propericiazine are required for a better understanding about its possible mechanisms that can induce the observed dose-response behavioral changes.

\section{References}

1. Ban OT (1969). Psychopharmacology. Williams \& Wilkins, Baltimore, MD, USA.

2. Anonymous (1967). Pericyazine. British Medical Journal, 1: 352-353.

3. Wiecek Z (1971). Attempts at treatment of children at a special institution with propericiazine. Psychiatria Polska, 5: 61-63.

4. Litinschi G, Fechervary E \& Ciumageanu D (1970). Some results of the use of propericiazine (RP8909) in the treatment of disorders of conduct and character in children and adolescents. Neurologia, Psihiatria, Neurochirurgia, 15: 493-504.

5. Takahashi M \& Akagi M (1996). Case report of sodium valproate treatment of aggression associated with Alzheimer's disease. No To Shinkei. Brain and Nerve, 48: 757-760.

6. Nishikawa T, Tanaka M, Tsuda A, Koga I \& Uchida Y (1989). Prophylactic effects of neuroleptics in symptom-free schizophrenics: a comparative dose-response study of haloperidol and propericiazine. Psychopharmacology, 82: 153-156

7. Jenner FA (1970). Pericyazine in clinical practice. Laval Medical, 41: 796-804.

8. Rodgers RJ, Johnson NJT, Champion AJ \& Mills S (1996). Modulation of plus-maze behaviour in mice by preferential $\mathrm{D}_{3}$-receptor agonist 7-OH-DPAT. Pharmacology, Biochemistry and Behavior, 54: 79-84.

9. Tischler B, Patriasz K, Beresford J \& Bunting R (1972). Experience with pericyazine in profoundly and severely retarded children. Canadian Medical Association Journal, 106: 136-141.
10. Rickels K \& Schweizer EE (1987). Current pharmacotherapy in anxiety and panic. In: Meltzer HY (Editor), Psychopharmacology: The Third Generation of Progress. Raven Press, New York, NY, USA, 1193-1203.

11. Pellow S, Chopin P, File SE \& Briley M (1985). Validation of openclosed arm entries in an elevated plus-maze as a measure of anxiety in the rat. Journal of Neuroscience Methods, 14: 149-176.

12. Salgueiro JB, Ardenghi $P$, Dias M, Ferreira MBC, Izquierdo I \& Medina JH (1997). Anxiolytic natural and synthetic flavonoid ligands of the central benzodiazepine receptor have no effect on memory tasks in rats. Pharmacology, Biochemistry and Behavior, 58: 1-5.

13. Quevedo J, Vianna MRM, Roesler R, De-Paris F, Izquierdo I \& Rose SPR (1999). Two time windows of anisomycin-induced amnesia for inhibitory avoidance training in rats: protection from amnesia by pretraining but not preexposure to the task apparatus. Learning and Memory, 6: 600-607.

14. Coward DM (1982). Classical and non-classical neuroleptics induce supersensitivity of nigral GABA-ergic mechanisms in rat. Psychopharmacology, 78: 180-184.

15. Marco E, Mao CC, Revueleta A, Peralta E \& Costa E (1978). Turnover rates of gamma-aminobutyric acid in substantia nigra, $N$. caudatus, globus pallidus and N. accumbens of rats injected with cataleptogenic and non-cataleptogenic antipsychotics. Neuropharmacology, 17: 589-596. 